\title{
SPANS OF SIMPLE TRIODS
}

\author{
THELMA WEST
}

(Communicated by Doug W. Curtis)

\begin{abstract}
We calculate and estimate the spans of objects in certain classes of continua; our main interest is in those objects with positive span.
\end{abstract}

In 1964, the concept of the span of a metric space was introduced by A. Lelek (see [2, p. 209]). Since that time, some modified versions of the span were considered (cf. [4 and 5]). To date the metric spaces for which the various spans have been explicitly calculated have mainly been objects for which the span is zero. It is still an unsettled problem whether or not continua of span zero are arc-like (see [3, p. 93]). In this paper, we calculate and estimate the spans of objects in certain classes of continua, and our main interest is in those objects with positive span.

Questions have been raised concerning the relationships between these various versions of spans. For instance, it was conjectured that the surjective span is always at least a half of the span (see [5, p. 37]). We answer this conjecture in the affirmative for some types of simple tirods (see 2.5). Another problem concerning simple triods asks if the semispan and the span are always equal (see [5, p. 38]). This problem is solved positively in $\S 2$ for some classes of simple triods.

In 1961, the width of a tree-like continuum was introduced by C. E. Burgess (see $[1, \mathrm{p} .447])$. The relationship of the width and the span is only vaguely known (cf. $[6$, p. 191]). We involve, in $\S 2$, the widths of simple triods in our estimations of the span (see 2.4, for example).

1. Preliminaries. All spaces studied in this paper are assumed to be nonempty metric spaces. The distance between two points $x, y$ of a metric space $X$ is denoted by $d(x, y)$. If $x \in X$ and $A, B \subset X$ are nonempty subsets, we write

$$
\begin{gathered}
d(x, A)=\operatorname{Inf}\{d(x, y): y \in A\}, \\
d(A, B)=\operatorname{Inf}\{d(x, y): x \in A, y \in B\} .
\end{gathered}
$$

If $a \in X$ and $\varepsilon>0$, the open ball and the closed ball, centered at the point $a$ with radius $\varepsilon$, are defined by the formulas

$$
\begin{aligned}
& B(a, \varepsilon)=\{x \in X: d(a, x)<\varepsilon\}, \\
& \bar{B}(a, \varepsilon)=\{x \in X: d(a, x) \leq \varepsilon\},
\end{aligned}
$$

respectively. Notice that the closure $\operatorname{cl} B(a, \varepsilon)$ is contained in $\bar{B}(a, \varepsilon)$, but the closed ball can be larger, as a set, then the closure of the open ball.

Received by the editors October 16, 1986.

1980 Mathematics Subject Classification (1985 Revision). Primary 54F15; Secondary 54F20. 
All mappings are assumed to be continuous functions. If $f: X \rightarrow Y$ and $g: X \rightarrow$ $Z$ are mappings, the diagonal mapping $f \Delta g: X \rightarrow Y \times Z$ into the product $Y \times Z$ is defined by the formula

$$
(f \Delta g)(x)=(f(x), g(x)) \quad(x \in X),
$$

so that $f \Delta g$ is continuous. The standard projections $p_{1}, p_{2}: X \times X \rightarrow X$ are mappings defined by $p_{1}(x, y)=x$ and $p_{2}(x, y)=y$ for $(x, y) \in X \times X$.

Let $X$ be a nonempty metric space. The surjective span $\sigma^{*}(X)$ of $X$ is the least upper bound of real numbers $\alpha$ such that there exist nonempty connected sets $C_{\alpha} \subset X \times X$ with $d(x, y) \geq \alpha$ for $(x, y) \in C_{\alpha}$ and

$$
p_{1}\left(C_{\alpha}\right)=p_{2}\left(C_{\alpha}\right)=X
$$

Relaxing condition $\left(\sigma^{*}\right)$ to the conditions

$$
\begin{gathered}
p_{1}\left(C_{\alpha}\right)=p_{2}\left(C_{\alpha}\right), \\
p_{1}\left(C_{\alpha}\right)=X, \\
p_{1}\left(C_{\alpha}\right) \subset p_{2}\left(C_{\alpha}\right),
\end{gathered}
$$

we obtain the definitions of the span $\sigma(X)$, the surjective semispan $\sigma_{0}^{*}(X)$, and the semispan $\sigma_{0}(X)$ of $X$, respectively.

By a continuum we mean a compact metric space which is connected.

1.1. If $X$ is a metric space and $\tau=\sigma^{*}, \sigma, \sigma_{0}^{*}, \sigma_{0}$, then $\tau(X)$ is the least upper bound of real numbers $\alpha$ such that there exist nonempty connected sets $C$ and continuous mappings $f, g: C \rightarrow X$ with $d[f(c), g(c)] \geq \alpha$ for $c \in C$ and

$\left(\sigma^{*}\right)^{\prime}$

$(\sigma)^{\prime}$

$\left(\sigma_{0}^{*}\right)^{\prime}$

$\left(\sigma_{0}\right)^{\prime}$

$$
\begin{gathered}
f(C)=g(C)=X, \\
f(C)=g(C), \\
g(C)=X, \\
f(C) \subset g(C),
\end{gathered}
$$

respectively. Moreover, if $X$ is a compact metric space, then $C$ then be assumed to be continua.

Proof. Observe that, given $C_{\alpha}$, then taking $C=C_{\alpha}, f=p_{1}$, and $g=p_{2}$, condition $(\tau)$ implies condition $(\tau)^{\prime}$. On the other hand, given $C, f$ and $g$, then taking $C_{\alpha}=(f \Delta g)(C)$, condition $(\tau)^{\prime}$ implies condition $(\tau)$. Moreover, if $X$ is a compact metric space and $C_{\alpha} \subset X \times X$ is a connected set satisfying $(\tau)$, then the closure $C=\operatorname{cl} C_{\alpha}$ also satisfies $(\tau)$, as well as the same requirement for the distances $d(x, y)$, and $C$ is a continuum.

1.2. If $X$ is a metric space, then

$$
\begin{aligned}
& 0 \leq \sigma^{*}(X) \leq \sigma(X) \leq \sigma_{0}(X) \leq \operatorname{diam} X \\
& 0 \leq \sigma^{*}(X) \leq \sigma_{0}^{*}(X) \leq \sigma_{0}(X) \leq \operatorname{diam} X
\end{aligned}
$$

(see [4, p. 207]). 
PROOF. This follows directly from the implications:

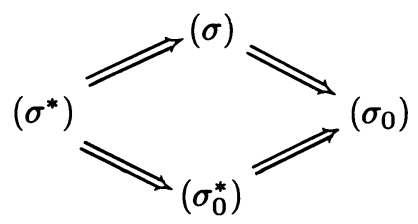

1.3. If $X$ is a metric space and $A \subset X$ is a nonempty subset, then $\sigma(A) \leq \sigma(X)$ and $\sigma_{0}(A) \leq \sigma_{0}(X)($ see $[4, p .207])$.

1.4. If $X$ is a metric space, $C$ is a continuum, and $f, g: C \rightarrow X$ are mappings satisfying condition $(\tau)^{\prime}$, where $\tau=\sigma^{*}, \sigma, \sigma_{0}^{*}, \sigma_{0}$, then there exists a point $c_{0} \in C$ such that $d\left[f\left(c_{0}\right), g\left(c_{0}\right)\right] \leq \tau(X)$.

ProOF. Suppose on the contrary that $d[f(c), g(c)]>\tau(X)$ for each $c \in C$. Since $C$ is compact, there is a number $\alpha_{0}>\tau(X)$ such that $d[f(c), g(c)] \geq \alpha_{0}$ for $c \in C$. This means that $\alpha_{0}$ is one of the numbers $\alpha$ appearing in the definition of $\tau(X)$, so that $\tau(X) \geq \alpha_{0}$, a contradiction.

By an arc we understand a space homeomorphic to the unit interval $[0,1]$ of the real line.

1.5. If $X$ is an arc, then $\sigma^{*}(X)=\sigma(X)=\sigma_{0}^{*}=\sigma_{0}(X)=0$ (see [5, p. 36]).

By a simple closed curve we understand a space homeomorphic to the unit circle $\left\{(u, v): u^{2}+v^{2}=1\right\}$ on the plane. A tree is a space homeomorphic to a connected one-dimensional polyhedron which contains no simple closed curve. A continuum $X$ is called tree-like (or arc like) provided there exists, for each $\varepsilon>0$, a finite open cover of $X$ whose members have diameters smaller than $\varepsilon$ and whose nerve, if nondegenerate, is a tree (or an arc, respectively). Finite open covers whose nerves are arcs are called chains. That is why arc-like continua are also called chainable. If $C$ is a collection of sets, we denote by $|C|$ the union of all members of $C$.

Let $X$ be a nonempty compact metric space. The width $w(X)$ of $X$ is the least upper bound of real numbers $\alpha$ which satisfy the following condition: there exists, for each $\varepsilon>0$, a finite open cover $C$ of $X$ such that each member of $C$ has diameter smaller than $\varepsilon$ and

$$
\left.\operatorname{Max}\left\{d(A),\left|C^{\prime}\right|\right): A \in C\right\} \geq \alpha
$$

for each chain $C^{\prime}$ contained in $C$. This definition of the width is slightly different from that of the width of a tree-like continuum, as given in [1], but it can be shown that, in the case of tree-like continua, the two definitions are equivalent (see [6, p. 184]).

1.6. If $X$ is a tree, then

$$
w(X)=\operatorname{Inf}\{\operatorname{Sup}\{d(x, A): x \in X\}: A \subset X, A \text { arc }\}
$$

(see [6, p. 186]).

By a simple triod we understand a space $T$ which admits a decomposition $T=$ $A_{0} \cup A_{1} \cup A_{2}$, where $A_{i}$ are arcs having a common endpoint $v$ and $A_{i} \cap A_{i+1}=\{v\}$ for $i=0,1,2$, and the subscripts of $A_{j}$ taken $\bmod 3$. Each simple triod is a tree. The arcs $A_{0}, A_{1}, A_{2}$ are called branches of the simple triod $T$, and the point $v$ is called the branch-point of $T$. 
1.7. If $T$ is a simple triod with branches $A_{0}, A_{1}, A_{2}$, then

$$
w(T)=\operatorname{Min}\left\{\operatorname{Max}\left\{d\left(x, A_{i+1} \cup A_{i+2}\right): x \in A_{i}\right\}: i=0,1,2\right\},
$$

where the subscripts are taken $\bmod 3$ (see [6, p. 187]).

1.8. If $T$ is a simple triod, then $w(T) \leq \sigma^{*}(T)$ (see $\left.[4, p .210 ; 7, p .208]\right)$.

For some simple triods, the weak inequality in 1.8 becomes the equality (cf. 3.1). However, in general, this is not true. It is known that there exists, for each $\varepsilon>0$, a simple triod $T$ on the plane such that $\sigma(T)=1$ and $w\left(T^{\prime}\right)<\varepsilon$ for each simple triod $T^{\prime}$ contained in $T$ (see [6, p. 195]). In such a triod $T$, a subtriod $T^{\prime}$ can be selected with $\sigma^{*}\left(T^{\prime}\right)=\sigma(T)$, whence $\sigma^{*}\left(T^{\prime}\right)=1$. Thus we can have simple triods with surjective span equal 1 and the width as small as one wishes.

2. Main results. The first theorem gives an estimate for the semispan of certain continua related to simple triods.

2.1. THEOREM. If $K$ and $L$ are continua such that $K \cap L=\{v\}, L \subset \bar{B}(v, \delta)$, where $\delta \geq 0$, and $\sigma_{0}(L) \leq \delta+\sigma_{0}(K)$, then $\sigma_{0}(K \cup L) \leq \delta+\sigma_{0}(K)$.

PROOF. Let $h: K \cup L \rightarrow K$ be the mapping defined by

$$
h(x)= \begin{cases}x & \text { if } x \in K, \\ v & \text { if } x \in L .\end{cases}
$$

Let $f, g: C \rightarrow K \cup L$ be mappings such that $C$ is a continuum and $f(C) \subset g(C)$. Considering the composites $h f, h g: C \rightarrow K$ we see, by 1.4 , that there exists a point $c \in C$ such that $d[h f(c), h g(c)] \leq \sigma_{0}(K)$. We distinguish six cases as follows.

Case 1. $f(c) \in K, g(c) \in K$. Then $h f(c)=f(c)$ and $h g(c)=g(c)$, whence

$$
d[f(c), g(c)]=d\left[h f(c), h g(c) \leq \sigma_{0}(K) \leq \delta+\sigma_{0}(K) .\right.
$$

Case 2. $f(c) \in K, g(c) \in L$. Then $h f(c)=f(c)$ and $h g(c)=v$, whence

$$
\begin{aligned}
d[f(c), g(c)] & \leq d[f(c), v]+d[v, g(c)] \leq d[f(c), v]+\delta \\
& =d[h f(c), h g(c)]+\delta \leq \sigma_{0}(K)+\delta .
\end{aligned}
$$

Case 3. $f(c) \in L, g(c) \in K$. Then $h f(c)=v$ and $h g(c)=g(c)$, whence

$$
\begin{aligned}
d[f(c), g(c)] & \leq d[f(c), v]+d[v, g(c) \leq \delta+d[v, g(c)] \\
& =\delta+d[h f(c), h g(c)] \leq \delta+\sigma_{0}(K) .
\end{aligned}
$$

Case 4. $f(c) \in L, g(c) \in L$ and

$$
(f \triangle g)(C) \cap[(L \times\{v\}) \cup(\{v\} \times L)]=\phi .
$$

In this case, we observe that the sets $A$ and $B$ defined by the formulas

$$
\begin{gathered}
A=(L \backslash\{v\}) \times(L \backslash\{v\}), \\
B=[(K \cup L) \times(K \backslash\{v\})] \cup[(K \backslash\{v\}) \times(K \cup L)]
\end{gathered}
$$

are disjoint open subsets of the product $(K \cup L) \times(K \cup L)$ and

$$
(f \triangle g)(C) \subset[(K \cup L) \times(K \cup L)] \backslash[(L \times\{v\}) \cup(\{v\} \times L)]=A \cup B,
$$

by (1). Also, the point $(f(c), g(c))$ belongs to $\left(f \Delta^{\circ} g\right)(C)$, and $f(c), g(c) \in L \backslash\{v\}$, by (1). Thus $(f(c), g(c)) \in A$ which, by the connectedness of $(f \triangle g)(C)$, implies 
that $(f \triangle g)(C) \subset A \subset L \times L$. Consequently, $f(C) \subset g(C) \subset L$, and, by 1.4 , there exists a point $c^{\prime} \in C$ such that

$$
d\left[f\left(c^{\prime}\right), g\left(c^{\prime}\right)\right] \leq \sigma_{0}(L) \leq \delta+\sigma_{0}(K) .
$$

Case 5. $(f \Delta g)(C) \cap(L \times\{v\}) \neq \varnothing$. Then there exist a point $c^{\prime \prime} \in C$ with $f\left(c^{\prime \prime}\right) \in L$ and $g\left(c^{\prime \prime}\right)=v$, whence

$$
d\left[f\left(c^{\prime \prime}\right), g\left(c^{\prime \prime}\right)\right]=d\left[f\left(c^{\prime \prime}\right) v\right] \leq \delta \leq \delta+\sigma_{0}(K) .
$$

Case 6. $(f \Delta g)(C) \cap(\{v\} \times L) \neq \varnothing$. Then there exists a point $c^{\prime \prime \prime} \in C$ with $f\left(c^{\prime \prime \prime}\right)=v$ and $g\left(c^{\prime \prime \prime}\right) \in L$, whence

$$
d\left[f\left(c^{\prime \prime \prime}\right), g\left(c^{\prime \prime \prime}\right)\right]=d\left[v, g\left(c^{\prime \prime \prime}\right)\right] \leq \delta \leq \delta+\sigma_{0}(K) .
$$

In all six cases, we have established the existence of a point $c \in C$ such that the distance $d[f(c), g(c)]$ is not greater than $\delta+\sigma_{0}(K)$. If all these distances are supposed to be not less than a number $\alpha \geq 0$, then $\alpha \leq \delta+\sigma_{0}(K)$. The semispan $\sigma_{0}(K \cup L)$ is, by 1.1 , the least upper bound of such $\alpha$ 's, and therefore

$$
\sigma_{0}(K \cup L) \leq \delta+\sigma_{0}(K)
$$

2.2. THEOREM. If $K$ and $L$ are continua such that $K \cap L=\{v\}, L \subset \bar{B}(v, \delta)$, where $\delta \geq 0$, and $\sigma(L) \leq \delta+\sigma(K)$, then $\sigma(K \cup L) \leq \delta+\sigma(K)$.

PROOF. The proof of 2.2 is a replica of that of 2.1. Everywhere in the proof of 2.1 the semispan $\sigma_{0}$ is to be replaced by the span $\sigma$, and the inclusion $f(C) \subset g(C)$ is to be replaced by the equality $f(C)=g(C)$.

2.3. THEOREM. If $T$ is a simple triod with a branch-point $v$ and $T^{\prime} \subset T$ is a simple triod such that one branch of $T$ is contained in $\bar{B}\left[v, w\left(T^{\prime}\right)\right]$, then

(i) $\sigma(T)=\sigma_{0}(T)=\sigma\left(T^{\prime}\right)=\sigma_{0}\left(T^{\prime}\right)=w\left(T^{\prime}\right)$,

(ii) $\sigma^{*}\left(T^{\prime}\right)=\sigma_{0}^{*}\left(T^{\prime}\right)=w\left(T^{\prime}\right)$.

ProOF. Let $L_{0}, L_{1}, L_{2}$ be the branches of $T$ with $L_{0} \subset \bar{B}\left[v, w\left(T^{\prime}\right)\right]$. Since $L_{0}$ and $L_{1} \cup L_{2}$ are arcs, we have $\sigma_{0}\left(L_{0}\right)=\sigma_{0}\left(L_{1} \cup L_{2}\right)=0$, by 1.5. Applying 2.1 to $K=L_{1} \cup L_{2}, L=L_{0}$ and $\delta=w\left(T^{\prime}\right)$, we obtain $\sigma_{0}(T) \leq w\left(T^{\prime}\right)$. Note that $w\left(T^{\prime}\right) \leq \sigma^{*}\left(T^{\prime}\right) \leq \sigma\left(T^{\prime}\right)$, by 1.2 and 1.8. Also, $T^{\prime} \subset T$ implies that $\sigma\left(T^{\prime}\right) \leq \sigma(T)$ and $\sigma_{0}\left(T^{\prime}\right) \leq \sigma_{0}(T)$, by 1.3 . Thus

$$
\begin{aligned}
& w\left(T^{\prime}\right) \leq \sigma\left(T^{\prime}\right) \leq \sigma(T) \leq \sigma_{0}(T) \leq w\left(T^{\prime}\right), \\
& w\left(T^{\prime}\right) \leq \sigma\left(T^{\prime}\right) \leq \sigma_{0}\left(T^{\prime}\right) \leq \sigma_{0}(T) \leq w\left(T^{\prime}\right)
\end{aligned}
$$

which yields (i). Hence $w\left(T^{\prime}\right) \leq \sigma^{*}\left(T^{\prime}\right) \leq \sigma\left(T^{\prime}\right)=w\left(T^{\prime}\right)$ and

$$
w\left(T^{\prime}\right) \leq \sigma^{*}\left(T^{\prime}\right) \leq \sigma_{0}^{*}\left(T^{\prime}\right) \leq \sigma_{0}\left(T^{\prime}\right)=w\left(T^{\prime}\right),
$$

by 1.2 . Consequently, (ii) is proved.

The next theorem gives an estimate for the surjective span of a simple triod in terms of the widths of its subtriods. Note that the width of a subtriod may be greater than the width of the triod (see 3.2). 
2.4. THEOREM. If $T^{\prime} \subset T$ are simple triods, then $\sigma^{*}(T) \geq w\left(T^{\prime}\right) / 2$.

PROOF. Let $p_{1}, p_{2}: T \times T \rightarrow T$ denote the standard projections of the product, that is, $p_{1}(x, y)=x$ and $p_{2}(x, y)=y$ for $(x, y) \in T \times T$. Let $\Phi: T \times T \rightarrow T \times T$ denote the coordinate-switch homeomorphism, that is, $\Phi(x, y)=(y, x)$ for $(x, y) \in T \times T$. We have $p_{2}=p_{1} \Phi$, and the composite $\Phi \Phi$ is the identity mapping of $T \times T$. Let

$$
T=L_{0} \cup L_{1} \cup L_{2}, \quad T^{\prime}=L_{0}^{\prime} \cup L_{1}^{\prime} \cup L_{2}^{\prime}
$$

be the decompositions of the triods $T$ and $T^{\prime}$ into branches $L_{i}^{\prime} \subset L_{i}(i=0,1,2)$. By 1.7 , there exist points $a_{i} \in L_{i}^{\prime}$ such that

$$
d\left(a_{i}, L_{i+1}^{\prime} \cup L_{i+2}^{\prime}\right) \geq w\left(T^{\prime}\right) \quad(i=0,1,2),
$$

where the subscripts are taken $\bmod 3$. Let $v$ denote the branch-point of $T$ (and also of $\left.T^{\prime}\right)$. Let $M_{i}$ be the subarc of the arc $L_{i}^{\prime}$ having end-points $v$ and $a_{i}(i=0,1,2)$. Hence

$$
d\left(a_{i}, M_{i+1} \cup M_{i+2}\right) \geq w\left(T^{\prime}\right) \quad(i=0,1,2) .
$$

Let $C$ be the subset of the product $T \times T$ defined by the formula

$$
\begin{aligned}
C= & {\left[\left\{a_{0}\right\} \times\left(M_{1} \cup M_{2}\right)\right] \cup\left[\left(M_{1} \cup M_{2}\right) \times\left\{a_{0}\right\}\right] } \\
& \cup\left[\left\{a_{1}\right\} \times\left(M_{0} \cup M_{2}\right)\right] \cup\left[\left(M_{0} \cup M_{2}\right) \times\left\{a_{1}\right\}\right] \\
& \cup\left[\left\{a_{2}\right\} \times\left(M_{0} \cup M_{1}\right)\right] \cup\left[\left(M_{0} \cup M_{1}\right) \times\left\{a_{2}\right\}\right],
\end{aligned}
$$

so that $C$ is the union of six connected sets. The first set meets both the fourth and the sixth sets. The second set meets the third and fifth sets. Also, the third and sixth sets meet. Therefore $C$ is connected. Let $M=M_{0} \cup M_{1} \cup M_{2}$. It follows from the definition of $C$ and from (2) that

$$
\begin{gathered}
p_{1}(C)=p_{2}(C)=M, \quad \Phi(C)=C, \\
d(x, y) \geq w\left(T^{\prime}\right) \quad \text { for }(x, y) \in C .
\end{gathered}
$$

Let $\varepsilon$ be an arbitrary number with $0<\varepsilon<w\left(T^{\prime}\right) / 2$. Let $L$ be a branch of $T$, that is, $L=L_{i}(i=0,1,2)$. We shall construct a connected subset $C(L)$ of the product $T \times T$ such that

$$
\begin{gathered}
C \cap C(L) \neq \varnothing, \\
L \backslash M \subset p_{1}[C(L)], \\
d(x, y)>w\left(T^{\prime}\right) / 2-\varepsilon \text { for }(x, y) \in C(L) .
\end{gathered}
$$

Consider the closed balls $B_{0}, B_{1}$ and $B_{2}$ in the simple triod $T$ defined by the formulas

$$
B_{j}=\bar{B}\left[a_{j}, w\left(T^{\prime}\right) / 2-\varepsilon\right] \quad(j=0,1,2) .
$$

According to (2), the centers of these balls are of distance greater than or equal to $w\left(T^{\prime}\right)$ from each other. Thus these three balls are pairwise disjoint closed subsets of $T$, and there exists a number $\eta>0$ such that if $x, y \in T$ and the points $x, y$ belong to two different balls $B_{0}, B_{1}, B_{2}$, then $d(x, y)>\eta$. Let $b_{i}$ denote the endpoint of the $\operatorname{arc} L=L_{i}$ different from $v$, so that $a_{i} \in L$ and $b_{i}$ may coincide with $a_{i}$, but not necessarily. We consider the arc $L$ as being ordered from its endpoint $v$ to the endpoint $b_{i}$. If $x, y \in L$, we denote by $[x, y]$ the subarc of $L$ with endpoints $x$ and 
$y$. We do not exclude degenerate subarcs, that is, the singletons $[x, x]=\{x\}$. Let us define a sequence $x_{1}, x_{2}, \ldots$ of points of $L$ as follows. Let $x_{1}=a_{i}$. Therefore $x_{1} \in B_{i}$. Suppose $x_{k}$ is defined $(k \geq 1)$ such that $x_{k} \in B_{j_{k}}$, where $j_{k}=0,1,2$. We let $x_{k+1}$ be the first point of $\left[x_{k}, b_{i}\right]$ belonging to the union of two other balls, that is, belonging to $B_{j_{k}+1} \cup B_{j_{k}+2}$ with subscripts taken mod 3 , if such points in $\left[x_{k}, b_{i}\right]$ exist. If they do not, $x_{k+1}$ is not defined and the sequence stops. Observe that if $x_{k+1}$ is defined, then the arc $\left[x_{k}, x_{k+1}\right]$ has endpoints in two different balls $B_{0}, B_{1}, B_{2}$, whence its diameter is greater than $\eta$. The $\operatorname{arc} L$ cannot contain an infinite chain of such arcs, and so, indeed, the sequence comes to a stop. In this way, we have defined an ordered finite sequence of points $x_{1}, x_{2}, \ldots, x_{n}$ of $L$. We have $x_{k} \in B_{j_{k}}$ for $k=1,2, \ldots, n$, and $j_{k} \neq j_{k+1}$ for $k=1,2, \ldots, n-1$. Also, $j_{1}=i$ and the last point $x_{n}$ may, but not necessarily, coincide with the endpoint $b_{i}$ of $L$.

The arc $\left[x_{k}, x_{k+1}\right]$ has endpoints in $B_{j_{k}}$ and $B_{j_{k+1}}$, respectively. Moreover, by the way it was selected, $\left[x_{k}, x_{k+1}\right]$ is disjoint with the third remaining ball $B_{3-\left(j_{k}+j_{k+1}\right)}$. We now define two sequences $D_{1}, \ldots, D_{n}$ and $E_{1}, \ldots, E_{n}$ of connected subsets of $T \times T$ by the formulas

$$
\begin{aligned}
& D_{k}=\left[x_{k}, x_{k+1}\right] \times\left\{a_{3-\left(j_{k}+j_{k+1}\right)}\right\} \quad(k=1, \ldots, n-1), \\
& D_{n}=\left[x_{n}, b_{i}\right] \times\left\{a_{j_{n}+1}\right\}, \\
& E_{k}=\left\{x_{k}\right\} \times\left(M_{j_{k}+1} \cup M_{j_{k}+2}\right) \quad(k=1, \ldots, n),
\end{aligned}
$$

where the subscripts are taken $\bmod 3$. The set $C(L)$ is defined to be the union

$$
C(L)=\left(D_{1} \cup \cdots \cup D_{n}\right) \cup\left(E_{1} \cup \cdots \cup E_{n}\right) .
$$

Let $l=3-\left(j_{k}+j_{k+1}\right)$, that is, $l$ is the number among $0,1,2$ which is different from both $j_{k}$ and $j_{k+1}$. Hence $l=j_{k}+1(\bmod 3)$ or $l=j_{k}+2(\bmod 3)$, and also $l=j_{k+1}+1(\bmod 3)$ or $l=j_{k+1}+2(\bmod 3)$. We see that the point $\left(x_{k}, a_{l}\right)$ belongs to both sets $D_{k}$ and $E_{k}$, and the point $\left(x_{k+1}, a_{l}\right)$ belongs to both sets $D_{k}$ and $E_{k+1}$ $(k=1, \ldots, n-1)$. Furthermore, $\left(x_{n}, a_{j_{n}+1}\right)$ belongs to both $D_{n}$ and $E_{n}$. Hence $D_{k} \cap E_{k} \neq \varnothing$ for $k=1, \ldots, n$ and $D_{k} \cap E_{k+1} \neq \varnothing$ for $k=1, \ldots, n-1$. It follows that the set $C(L)$ is connected. Since $x_{1}=a_{i}$, the set $E_{1}$ is $\left\{a_{i}\right\} \times\left(M_{j_{k}+1} \cup M_{j_{k}+2}\right)$, and so it is either the first or the third or the fifth set appearing in the definition of $C$, which implies (5). Inclusion (6) is also satisfied because

$$
\begin{aligned}
L \backslash M & \subset\left[a_{i}, b_{i}\right]=\left[x_{1}, b_{i}\right]=\bigcup_{k=1}^{n-1}\left[x_{k}, x_{k+1}\right] \cup\left[x_{n}, b_{i}\right] \\
& =\bigcup_{k=1}^{n-1} p_{1}\left(D_{k}\right) \cup p_{1}\left(D_{n}\right)=p_{1} \bigcup_{k=1}^{n} D_{k} \subset p_{1}[C(L)] .
\end{aligned}
$$

To prove (7), let us take a point $(x, y) \in C(L)$. If $(x, y) \in D_{k}(k=1, \ldots, n-1)$, then $x \in\left[x_{k}, x_{k+1}\right]$ and $y=a_{l}$. We have observed that the arc $\left[x_{k}, x_{k+1}\right]$ is disjoint with the closed ball $B_{l}$ which is centered at $a_{l}$. Thus

$$
d(x, y)=d\left(x, a_{l}\right)>w\left(T^{\prime}\right) / 2-\varepsilon .
$$

If $(x, y) \in D_{n}$, then $x \in\left[x_{n}, b_{i}\right]$ and $y=a_{j_{n}+1}$. But $x_{n} \in B_{j_{n}}$, and $x_{n+1}$ does not exist meaning that the arc $\left[x_{n}, b_{i}\right]$ is disjoint with $B_{j_{n}+1} \cup B_{j_{n}+2}$; hence, 
in particular, it is disjoint with the closed ball $B_{j_{n}+1}$ which is centered at $a_{j_{n}+1}$. Thus

$$
d(x, y)=d\left(x, a_{j_{n}+1}\right)>w\left(T^{\prime}\right) / 2-\varepsilon .
$$

If $(x, y) \in E_{k}(k=1, \ldots, n)$, then $x=x_{k}$ and $y \in M_{j_{k}+1} \cup M_{j_{k}+2}$. But $x_{k} \in B_{j_{k}}$, where the closed ball $B_{j_{k}}$ is centered at $a_{j_{k}}$, and we use (2) to infer that

$$
\begin{aligned}
d(x, y) & =d\left(x_{k}, y\right) \geq d\left(a_{j_{k}}, y\right)-d\left(a_{j_{k}}, x_{k}\right) \\
& \geq w\left(T^{\prime}\right)-\left[w\left(T^{\prime}\right) / 2-\varepsilon\right] \\
& =w\left(T^{\prime}\right) / 2+\varepsilon>w\left(T^{\prime}\right) / 2-\varepsilon .
\end{aligned}
$$

This concludes the proof of (7). To end the proof of the theorem, consider the set

$$
F=C \cup \bigcup_{L=L_{0}, L_{1}, L_{2}} C(L) .
$$

Since $C$ and $C(L)$ are connected, it follows from (5) that $F$ is connected. Moreover, we have

$$
\begin{gathered}
T=L_{0} \cup L_{1} \cup L_{2}=M \cup \bigcup_{L=L_{0}, L_{1}, L_{2}}(L \backslash M) \\
\subset p_{1}(C) \cup \bigcup_{L=L_{0}, L_{1}, L_{2}} p_{1}[C(L)]=p_{1}(F),
\end{gathered}
$$

by (3) and (6). By (4) and (7), we obtain the inequality $d(x, y)>w\left(T^{\prime}\right) / 2-\varepsilon$ for each $(x, y) \in F$. Also $\varnothing \neq C \subset F$ and $C=\Phi(C) \subset \Phi(F)$, by (3). Consequently, the set $G=F \cup \Phi(F)$ is a connected subset of $T \times T$ and

$$
\begin{gathered}
T \subset p_{1}(F) \subset p_{1}(G) \subset T \\
T \subset p_{1}(F)=p_{1} \Phi \Phi(F)=p_{2} \Phi(F) \subset p_{2}(G) \subset T,
\end{gathered}
$$

which yields $p_{1}(G)=p_{2}(G)=T$. Finally, the inequality $d(x, y)>w\left(T^{\prime}\right) / 2-\varepsilon$ is clearly true for each $(x, y) \in G$ as well. The surjective span $\sigma^{*}(T)$ must then be not less than $w\left(T^{\prime}\right) / 2-\varepsilon$, and since $\varepsilon$ was allowed to be an arbitrarily small positive number, the conclusion of 2.4 follows.

2.5. COROLLARY. If $T$ is a simple triod with a branch-point $v$ and $T^{\prime} \subset T$ is a simple triod such that one branch of $T$ is contained in $\bar{B}\left[v, w\left(T^{\prime}\right)\right]$, then $\sigma(T) \leq$ $2 \sigma^{*}(T)$.

To get 2.5, apply 2.3 and 2.4. The simplest example of a triod shows that the weak inequalities in both 2.4 and 2.5 cannot be replaced by the equalities (see 3.1). Combining 2.3 and 2.4, we obtain estimates for the values of all spans of $T$ with the exception of $\sigma_{0}^{*}(T)$.

3. Examples. We give some examples of simple triods in the Euclidean 2-space $R^{2}$ with the ordinary Pythagorean distance $d$.

3.1. EXAMPLE. There exists a simple triod $T$ in $R^{2}$ such that $T \subset \bar{B}[v, w(T)]$, where $v$ is the branch-point of $T$, and

$$
\sigma(T)=\sigma_{0}(T)=\sigma^{*}(T)=\sigma_{0}^{*}(T)=w(T)=1 .
$$


Let $v=(0,0)$ be the origin in $R^{2}$. For two points $a, b \in R^{2}$, we denote by $\overline{a b}$ the straight line interval having $a$ and $b$ as endpoints. We take $a_{0}=(-1,0), a_{1}=(0,1)$, $a_{2}=(1,0)$, and $A_{i}=\overline{a_{i} v}(i=0,1,2)$. Let $T=A_{0} \cup A_{1} \cup A_{2}$.

We observe that $d\left(a_{i}, A_{i+1} \cup A_{i+2}\right)=1$ and $d\left(x, A_{i+1} \cup A_{i+2}\right)<1$ for $x \in$ $A-\left\{a_{i}\right\}$ and for $i=0,1,2$. Applying 1.7, we see that $w(T)=1$. From 1.8, we see that $\sigma^{*}(T) \geq 1$. To show the desired equality, by 1.2 , we just need to show that $\sigma_{0}(T) \leq 1$.

Let $C \subset T \times T$ be a nonempty connected set such that $p_{1}(C) \subset p_{2}(C)$, where $p_{1}, p_{2}: T \times T \rightarrow T$ are the standard projections.' Then $p_{2}(C)$ is a connected subset of $T$. The vertex $v$ cuts the simple triod $T$ into three components which are subsets of the three polygonal arcs forming $T$. If $v \notin p_{2}(C)$, the connected set $p_{2}(C)$ lies on one of the arcs and so does $p_{1}(C)$. The semispan of each arc is zero, by 1.5 , whence $C$ contains points $\left(q, q^{\prime}\right)$, with $d\left(q, q^{\prime}\right)$ arbitrarily small; in particular $d\left(q, q^{\prime}\right) \leq 1$ for some $\left(q, q^{\prime}\right) \in C$. If $v \in p_{2}(C)$, then there exists a point $q_{0} \in T$ such that $\left(q_{0}, v\right) \in C$ and $d\left(q_{0}, v\right) \leq 1$. In both cases, there does not exist a number $\alpha>1$ such that $d\left(q, q^{\prime}\right) \geq \alpha$ for $\left(q, q^{\prime}\right) \in C$. Consequently, $\sigma_{0}(T) \leq 1$.

3.2. EXAMPLE. There exists, for each number $\varepsilon$ with $0<\varepsilon<1$, a simple triod $T^{\varepsilon}$ in $R^{2}$ such that $T \subset T^{\varepsilon}$, where $T$ is the triod from 3.1 , and $w\left(T^{\varepsilon}\right)=\varepsilon$, while $w(T)=1$.

Let $T^{\varepsilon}=T \cup B^{\prime}$, where $b=(-\varepsilon, \varepsilon), c=(-\varepsilon, 1)$ and $B^{\prime}=\overline{a_{0} b} \cup \overline{b c}$. We see that the three branches of $T^{\varepsilon}$ are $A_{0} \cup B^{\prime}, A_{1}$, and $A_{2}$.

To determine the value of $\operatorname{Max}\left\{d\left(x, A_{0} \cup B^{\prime} \cup A_{2}\right): x \in A_{1}\right\}$ we distinguish two cases as follows.

Case 1. $x=(0, y)$, where $0 \leq y \leq \varepsilon$.

In this case $d\left(x, A_{0} \cup B^{\prime} \cup \overline{A_{2}}\right) \leq \varepsilon$, since $v \in A_{0} \cup B^{\prime} \cup A_{2}$ and $d(x, v) \leq \varepsilon$.

Case 2. $x=(0, y)$, where $\varepsilon<y \leq 1$.

In this case, $d\left(x, A_{0} \cup B^{\prime} \cup A_{2}\right)=\varepsilon$ since $(-\varepsilon, y) \in B^{\prime}, d[x,(-\varepsilon, y)]=\varepsilon$, and for each $z \in\left(A_{0} \cup B^{\prime} \cup A_{2}\right)-\{(-\varepsilon, y)\}, d(x, z)>\varepsilon$.

Hence, we get that $\operatorname{Max}\left\{d\left(x, A_{0} \cup B^{\prime} \cup A_{2}\right): x \in A_{1}\right\}=\varepsilon$. Since $d\left(a_{0}, A_{1} \cup A_{2}\right)=1$ and $d\left[a_{2},\left(A_{0} \cup B^{\prime}\right) \cup A_{1}\right]=1$, we see that $\operatorname{Max}\left\{d\left(x, A_{1} \cup A_{2}\right): x \in A_{0} \cup B^{\prime}\right\} \leq 1$ and $\operatorname{Max}\left\{d\left(x, A_{0} \cup B^{\prime} \cup A_{1}\right): x \in A_{2}\right\} \geq 1$. From 1.7, we conclude that $w\left(T^{\varepsilon}\right)=\varepsilon$.

ACKNOWLEDGMENT. Research supported in part by the John B. O'Hara Chemical Sciences Institute, University of Dallas.

\section{BIBLIOGRAPHY}

1. C. E. Burgess, Collections and sequences of continua in the plane. II, Pacific J. Math. 11 (1961), 447-454.

2. A. Lelek, Disjoint mappings and the span of spaces, Fund. Math. 55 (1964), 199-214.

3. __ Some problems concerning curves, Colloq. Math. 23 (1971), 93-98.

4. __ An example of a simple triod with surjective span smaller than span, Pacific J. Math. 64 (1976), 207-215.

5. __ On the surjective span and the semispan of connected metric spaces, Colloq. Math. 37 (1977), 35-45.

6. The span and the width of continua, Fund. Math. 98 (1978), 181-199.

7. A. Lelek and L. Mohler, Real-valued continuous functions and the span of continua, Colloq. Math. 32 (1975), 207-209.

DEPARTMENT OF MATHEMATICS, UNIVERSity OF SOUTHWESTERN Louisiana, LAFYETTE, LOUISIANA 70504 